\title{
Stability considerations of permanent magnet quadrupoles for CESR phase-III upgrade
}

\author{
W. Lou, D. Hartill, D. Rice, D. Rubin, and J. Welch \\ Laboratory of Nuclear Studies, Cornell University, Ithaca, New York 14853
}

(Received 5 March 1998; published 26 June 1998)

\begin{abstract}
The Cornell electron storage ring (CESR) phase-III upgrade plan includes very strong permanent magnet quadrupoles in front of the cryostat for the superconducting quadrupoles and physically as close as possible to the interaction point. Together with the superconducting quadrupoles, they provide tighter vertical focusing at the interaction point. The quadrupoles are built with neodymium iron boron $(\mathrm{NdFeB})$ material and operate inside the $15 \mathrm{kG}$ solenoid field. Requirements on the field quality and stability of these quadrupoles are discussed and test results are presented. [S1098-4402(98)00006-8]
\end{abstract}

PACS numbers: $75.50 . \mathrm{Ww}$

\section{INTRODUCTION}

The Cornell electron storage ring (CESR) phase-III luminosity upgrade plan includes very high gradient, $27.6 \mathrm{~cm}$ long, permanent magnet quadrupoles (PMQs) followed by a pair of superconducting quadrupoles [1]. These permanent magnet quadrupoles are located $33.7 \mathrm{~cm}$ away from the interaction point. This quadrupole scheme of the interaction region permits reduction of beta function at the interaction point $\beta_{v}^{*}$ to $1 \mathrm{~cm}$ or less and also helps to reduce the vertical beta function at the first parasitic interaction point, which occurs $2.1 \mathrm{~m}$ from the interaction point, as well as the peak vertical beta, which reduces aperture requirements and vertical chromaticity. By reducing the effects of parasitic crossings adjacent to the interaction point, it allows the operation with bunch spacing of $14 \mathrm{~ns}$. The luminosity after the upgrade will be increased dramatically due to the extra beam current implied by $9 \times 5$ bunches made possible by reducing the bunch spacing and the tighter vertical focusing at the interaction point [2]. Although it is designed for flat-beam crossing angle collision operation, the phase-III interaction region (IR) quadrupole scheme is compatible with the possible round beam configuration [3].

\section{PERMANENT MAGNET QUADRUPOLE CONSTRUCTION}

The permanent magnet quadrupole is made of three $9.2 \mathrm{~cm}$ long sections which are assembled individually then bolted together for the full quadrupole. The whole assembly is mounted to the drift chamber of the CLEO detector. The cross section view of the quadrupole assembly is shown in Fig. 1.

The permanent magnet quadrupoles extend from 337 to $616 \mathrm{~mm}$ from the interaction point and have a constant inner radius of $3.35 \mathrm{~cm}$ and a two-step outer radius. The dimensions of the quadrupole are chosen in such a way that the strength of the PMQ is as strong as possible and as physically close as possible to the interaction point in the limited space available for the PMQs. They will operate inside the $15 \mathrm{kG}$ solenoid field of the experimen- tal detector. They are built with neodymium iron boron $(\mathrm{NdFeB})$ material because of its lower price, higher commercially available remnant field $B_{r}$, and intrinsic coercivity $H_{c i}$ compared with samarium cobalt (SmCo) material. The quadrupoles are built with 16 azimuthal segments.

Magnet pole pieces were ordered with three easy-axis orientations $\left(0^{\circ}, 45^{\circ}\right.$, and $\left.90^{\circ}\right)$ and magnetized as shown in Fig. 1. The focusing strength of a permanent magnet quad is influenced by remnant field $\left(B_{r}\right)$, inner "pole-tip" radius $\left(r_{i}\right)$, and the ratio of outer to inner radius $\left(r_{o} / r_{i}\right)$. This last parameter, $r_{o} / r_{i}$, must be limited to avoid subjecting parts of the magnetic material to excessive demagnetizing fields, which could seriously degrade field quality. According to the numerical analysis with the Pandira code [4] and reverse magnetic field knock-down test with the magnet material, we find that the $r_{o} / r_{i}$ of 2.1 is appropriate to the magnet material with $H_{c i}$ of $21 \mathrm{kOe}$ and $B_{r}$ of $12 \mathrm{kG}$.

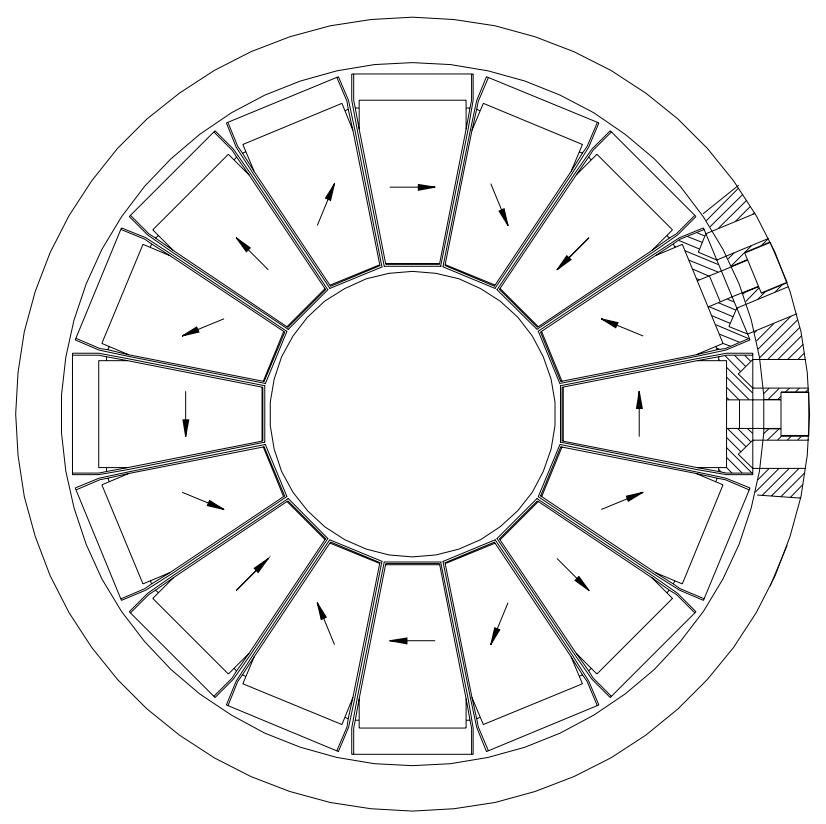

FIG. 1. Phase-III permanent magnet quadrupole cross section view. 
The magnet pole pieces were supplied by Magnet Sales and Manufacturing, Inc. The material is Shin-Etsu 36SH neodymium iron boron. The typical magnetic and mechanical characteristics of this material are listed in Table I.

The mechanical assembly of the quadrupole is similar to the existing rare-earth PMQs operating in the storage ring [5-8]. The permanent magnet pole pieces are affixed to a stainless backing plate using high temperature adhesive and covered by a stainless steel skin spot welded to the backing plate for extra protection. The backing plate with magnet was then screwed fast to the support shell. The weight of each quadrupole assembly is about $45 \mathrm{~kg}$. The measured quadrupole strength at $5.289 \mathrm{GeV}$ is listed in Table II.

\section{FIELD QUALITY AND STABILITY}

Both the quality and magnitude of the interaction region quadrupole magnetic field are critical to storage ring operation. The field quality determines nonlinear properties such as dynamic aperture and the field magnitude must be stable to avoid uncontrolled change in the betatron tune and optics functions of the storage ring. Many factors can affect the magnet and tend to alter the magnetic flux, which would change the field quality and quadrupole strength in our application. We have studied these influences and made predications for the resulting changes in magnetic flux. Many of these changes are one-time aging effects and can be minimized by first exposing the magnet to these influences and then retuning the magnet before placing it in service.

\section{A. Resistance to the irreversible demagnetization}

The permanent magnet maintains its magnetic flux because there are many small magnetic domains aligned by crystal anisotropy. A very high external magnetic field tends to disturb the domain alignment. When the magnet pole pieces are assembled into the quadrupole, some regions of the magnet material operate in a very strong antiparallel magnet field. For the case of our design, we find that this antiparallel magnet field could be as high as $13 \mathrm{kG}$ according to the Pandira simulation. Several different magnet samples were tested to determine the knock-down ratio of the remnant field $B_{r}$ with applied reverse external field. The knock-down ratio is defined as the magnetization change before and after the exposure of the external field divided by its original magnetization. The intrinsic coercivity of $21 \mathrm{kOe}$ was sufficient to limit the amount of demagnetization to a few percent with $13 \mathrm{kG}$ reverse external field. Figure 2 shows the amount of demagnetization vs the reverse external field for the $\mathrm{NdFeB} 36 \mathrm{SH}$ material we used. The material we are using could sustain $15 \mathrm{kG}$ reverse external field.

The quadrupole magnet will operate immersed in a $15 \mathrm{kG}$ axial solenoid field (perpendicular to the $\mathrm{NdFeB}$ permanent magnet easy axes) of the experimental detector. Several magnet pole pieces were exposed to an external $20 \mathrm{kG}$ perpendicular field. No demagnetization was found after the exposure.

\section{B. Temperature stability}

The properties of the magnet material change with temperature and time. The magnet can be stabilized by heating it to a temperature well above the operating temperature. This process speeds up the initial aging and slows down the rate of change thereafter.

All magnet pole pieces were thermally stabilized for $3 \mathrm{~h}$ at $100{ }^{\circ} \mathrm{C}$, the maximum temperature without irreversible loss of coercive force. The losses of magnetization during this procedure were measured to be less than $1 \%$ of the original magnetic moment. Several thermally stabilized magnet sample pieces were remeasured after being stored on the shelf for eight months and no magnetization changes were found.

It is also important that heat stabilization be performed when the magnets are in a field similar to that which they will see during operation. We performed the temperature stabilization process for the assembled quadrupole. Since the quadrupole is operated at room temperature, it is reasonable to do the temperature stabilization process for the quadrupole assembly at $70{ }^{\circ} \mathrm{C}$ for two hours. The quadrupole strength is decreased very slightly (less than $0.5 \%$ ) since the magnet material has very high intrinsic coercivity and all the magnet pieces have been individually heat stabilized to $100{ }^{\circ} \mathrm{C}$.

TABLE I. Typical characteristics of NdFeB $36 \mathrm{SH}$.

\begin{tabular}{lcc}
\hline \hline & cgs unit & SI unit \\
\hline Remnant field $B_{r}$ & $12.2 \mathrm{kG}$ & $1.22 \mathrm{~T}$ \\
Coercive force $H_{c}$ & $11.7 \mathrm{kOe}$ & $0.93 \times 10^{6} \mathrm{~A}$-turn $/ \mathrm{m}$ \\
Intrinsic coercive force $H_{c i}$ & $23 \mathrm{kOe}$ & $1.83 \times 10^{6} \mathrm{~A}$-turn $/ \mathrm{m}$ \\
Maximum energy & $36 \mathrm{MGOe}$ & $286 \mathrm{~kJ} / \mathrm{m}^{3}$ \\
Recoil permeability & 1.05 & 1.05 \\
Density & $7.5 \mathrm{~g} / \mathrm{cm}^{3}$ & $7.5 \times 10^{3} \mathrm{~kg} / \mathrm{m}^{3}$ \\
Electric resistivity & $2.0 \times 10^{-4} \Omega \cdot \mathrm{cm}$ & $2.0 \times 10^{-6} \Omega \cdot \mathrm{m}$ \\
Temperature coefficient of $B_{r}$ & $-0.1 \% /{ }^{\circ} \mathrm{C}$ & $-0.1 \% /{ }^{\circ} \mathrm{C}$ \\
Curie temperature & $310{ }^{\circ} \mathrm{C}$ & $310{ }^{\circ} \mathrm{C}$ \\
\hline \hline
\end{tabular}


TABLE II. Measured quadrupole magnetic strength (each section is $9.2 \mathrm{~cm}$ long).

\begin{tabular}{lccccc}
\hline \hline & $r_{i}$ & $r_{o}$ & Pole field at $r_{i}$ & Integrated strength & $\mathrm{k}$ \\
\hline & $\mathrm{cm}$ & $\mathrm{cm}$ & $\mathrm{kG}$ & $\mathrm{kG}-\mathrm{cm} / \mathrm{cm}$ & $\mathrm{m}^{-2}$ \\
Front section & 3.35 & 6.40 & 9.2 & 25.3 & 1.55 \\
Outer sections & 3.35 & 7.04 & 10.0 & 27.5 & 1.70 \\
\hline \hline
\end{tabular}

Since the Curie temperature of the $\mathrm{NdFeB}$ material is much lower than for the SmCo material used in the CESR's present IR quadrupoles, the temperature coefficient of $B_{r}$ was measured to be almost 3 times higher. This means that the quadrupole strength is also almost 3 times more sensitive to the temperature. Fortunately, the phase-III quadrupole's focal length is longer (weaker focusing) due to its shorter physical length, and the beta functions are lower, making the machine optics much less sensitive to changes in quadrupole strength. The quadrupole temperature should be controlled to within $0.2{ }^{\circ} \mathrm{C}$ to limit the storage ring vertical tune shift within 0.0005 integer. The temperature of the quadrupole will be controlled by running coolant through a $1 / 4$ in. tubing on the outer shell to remove heat from the silicon detector electronics, the most important heat source affecting the PMQs.

\section{Corrosion and surface oxidation}

$\mathrm{NdFeB}$ material is more subject to oxidation and corrosion than the SmCo material. All surfaces of the magnet piece were coated with cadmium chromate for corrosion and oxidation resistance.

\section{Radiation damage}

There are several reported studies about the radiation damage due to the neutrons and photons ( $\gamma$ and $\mathrm{x}$ rays) [9-12]. The data suggested that the integrated neutron fluences of $5 \times 10^{14} \mathrm{n} / \mathrm{cm}^{2}$ and $50 \mathrm{MR}$ of bremsstrahlung radiation is needed to show sizable damage to the $\mathrm{NdFeB}$ material. The radiation level in the

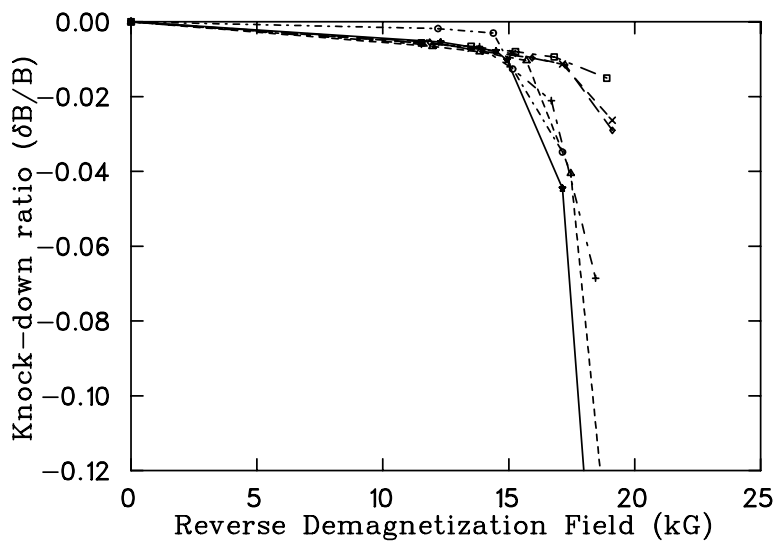

FIG. 2. Knock-down ratio vs the applied external reversal field for several magnet pieces. electron/positron storage rings is due mainly to the gamma bremsstrahlung and the synchrotron radiation. For CESR, it is estimated that the integrated dose of photons in the interaction region where the PMQs are located is a few MR for five years of operation. The present CESR neutron level is measured at about $2 \times 10^{9} \mathrm{n} / \mathrm{cm}^{2}$ per year. Both neutron and photon radiation levels for the five years of CESR phase-III operation are estimated to be much lower than the threshold level to show significant radiation damage. Several magnet sample pieces were exposed to a dose of $6 \mathrm{MR}$ of gamma radiation in a cobalt60 chamber and no magnetization change was found after the exposure.

\section{E. Field quality}

The effect of nonlinearities in the permanent magnet quadrupoles is evaluated by a tracking study. The dynamic aperture is computed for trajectories with initial energy offset 0,5 , and $10 \sigma_{E} / E$ for both the error free machine and a machine with errors. We find that a field error of less than $1 \times 10^{-3}$ deviation from a pure quadrupole field at a radius of $2.8 \mathrm{~cm}$ is required.

The multipole field could be caused by magnet pieceto-piece variations in magnetization, errors in positioning the magnet, and the antiparallel demagnetization by the local field observed by some of the magnet pieces when the magnets are assembled into the quadrupole.

The variation of $B_{r}$ and magnetization angle error of all of the magnet pieces were measured. We found that the $B_{r}$ variation is less than $\pm 2 \%$ and the angle error of the easy axis is within $2^{\circ}$. The field error is reduced by matching magnets with similar properties and a tuning procedure to adjust a small amount of radial motion of each magnet piece to cancel the measured multipole field. A rotating coil measurement system was built to measure the multipole field to the accuracy of 1 part in $10^{4}$ of quadrupole field at radius of $2.8 \mathrm{~cm}$. Measured results show that a quadrupole field with multipole field errors of $1 \times 10^{-3}$ at $2.8 \mathrm{~cm}$ radius was achieved.

\section{ACKNOWLEDGMENTS}

The authors thank J. Greenly and the Laboratory of Plasma Studies at Cornell for providing and helping with knock-down test equipment. Many thanks are due to T. Vandermark and R. Rice for helping with the tests and assembly. Special thanks are given to J. Cherwinka 
for helpful discussions. This work was supported by the National Science Foundation.

[1] J. Welch, G. Dugan, E. Nordberg, and D. Rice, Cornell Collide Beam Note, CBN97-21 (1997).

[2] D. Rubin, in Proceedings of the 1995 IEEE Particle Accelerator Conference, Dallas, Texas (IEEE, New York, 1995), p. 481.

[3] R. Talman, Phys. Rev. Lett. 74, 1590 (1995).

[4] Los Alamos Accelerator code Group, Poisson/Superfish Reference Manual, LA-UR-87-126 (1987).

[5] S. Herb, in Proceedings of the 1987 IEEE Particle Accelerator Conference, Washington, DC (IEEE,
New York, 1987), p. 1434.

[6] S. Herb, Cornell Collide Beam Note, CBN85-9 (1985).

[7] K. Halbach, Nucl. Instrum. Methods Phys. Res. 169, 1 (1980).

[8] K. Halbach, Nucl. Instrum. Methods Phys. Res. 198, 213 (1982).

[9] H. B. Luna and X. K. Maruyama, Nucl. Instrum. Methods Phys. Res., Sect. A 285, 349 (1989).

[10] W. V. Hassenzahl, T. M. Jenkins, Y. Namito, W. R. Nelson, and W.P. Swanson, Nucl. Instrum. Methods Phys. Res., Sect. A 291, 378 (1990).

[11] J. Pfluger and G. Heintze, Rev. Sci. Instrum. 66, 1946 (1995).

[12] J. R. Cost, R. D. Brown, A. L. Giorgi, and J. T. Stanley, Mat. Res. Soc. Symp. Proc. 96, 321 (1987). 